\title{
Testing for mean and correlation changes in microarray experiments: an application for pathway analysis
}

Mayer Alvo', Zhongzhu Liu', Andrew Williams ${ }^{2 *}$, Carole Yauk²

\begin{abstract}
Background: Microarray experiments examine the change in transcript levels of tens of thousands of genes simultaneously. To derive meaningful data, biologists investigate the response of genes within specific pathways. Pathways are comprised of genes that interact to carry out a particular biological function. Existing methods for analyzing pathways focus on detecting changes in the mean or over-representation of the number of differentially expressed genes relative to the total of genes within the pathway. The issue of how to incorporate the influence of correlation among the genes is not generally addressed.

Results: In this paper, we propose a non-parametric rank test for analyzing pathways that takes into account the correlation among the genes and compared two existing methods, Global and Gene Set Enrichment Analysis (GSEA), using two publicly available data sets. A simulation study was conducted to demonstrate the advantage of the rank test method.

Conclusions: The data indicate the advantages of the rank test. The method can distinguish significant changes in pathways due to either correlations or changes in the mean or both. From the simulation study the rank test out performed Global and GSEA. The greatest gain in performance was for the sample size case which makes the application of the rank test ideal for microarray experiments.
\end{abstract}

\section{Background}

DNA microarrays are powerful tools used for the analysis of genome-wide gene expression. The dimensionality of commercially available platforms has dramatically increased over the years. The technology has evolved rapidly and now provides a relatively accurate method to determine what genes are differentially regulated as a result of a particular condition. Although the technology is intended to provide a means to understand the response of a system as a whole, the interpretation of DNA microarray data has generally been carried out by analysis of individual genes for differential expression. With the broad goal of understanding the biology of the system, the evaluation of single genes is impractical.

Reducing the dimensionality of microarray data through the analysis of pathways or gene sets related to

\footnotetext{
* Correspondence: Andrew_Williams@hc-sc.gc.ca

2Environmental Health Science and Research Bureau, Environmental and Radiation Health Sciences Directorate, Health Canada, Ottawa ON K1A OK9
} Canada

(c) 2010 Alvo et al; licensee BioMed Central Ltd. This is an Open Access article distributed under the terms of the Creative Commons Attribution License (http://creativecommons.org/licenses/by/2.0), which permits unrestricted use, distribution, and reproduction in any medium, provided the original work is properly cited. biological functions, instead of analysing individual genes, will facilitate deriving biologically meaningful experimental results. However, classical multivariate approaches are generally not appropriate statistical tools for the analysis of pathways because the numbers of samples in microarray experiments are often very small, generally ranging from three to ten per experimental condition. As such, it is difficult to ascertain the nature of the underlying distribution. In 2002, an approach using Gene Ontology (GO) was proposed that assigns genes into groups and looks for over-representation of differentially expressed genes within these sets $[1,2]$. Since that time over 20 such tools have been developed [3-10].

The Fisher's Exact Test is one of the most popular methods underlying most software investigating overrepresentation of genes from a gene list for pathways, terms or ontologies. However, the assumption that the probes within pathways are independent is not satisfied since genes within pathways are highly associated. 
Moreover, an over-representation approach, such as the Fisher's Exact Test, focuses only on the number of significantly expressed probes, but ignores the magnitude of changes of the fluorescence intensity.

The Gene set enrichment analysis (GSEA) [5] method is becoming more commonly used for pathway analysis. This technique, introduced by Moothe et al. [4] involves the application of GSEA to pre-determined gene sets to identify differences in expression between normal and diseased patients. The methodology was later modified by Subrammanian et al [5]. GSEA consists of ranking the genes on the microarray, $g_{1}, g_{2}, \ldots, g_{M}$, by their signal-to-noise ratio(SNR),

$$
S N R=\frac{\left|\hat{\mu}_{1}-\hat{\mu}_{2}\right|}{\hat{\sigma}_{1}+\hat{\sigma}_{2}}
$$

Where $\hat{\mu}_{i}$ and $\hat{\sigma}_{i}$ are the estimated mean and standard deviations of normalized signal intensity for sample $i, i=1,2$.

Two empirical cumulative distribution functions are then calculated for each gene set, $G$ as follows,

$$
\begin{aligned}
& P_{H i t}\left(G, g_{i}\right)=\frac{\#\left[g_{j} \in G, 1 \leq j \leq i\right]}{N_{G}} \\
& P_{\text {Miss }}\left(G, g_{i}\right)=\frac{\#\left[g_{j} \notin G, 1 \leq j \leq i\right]}{M-N_{G}}
\end{aligned}
$$

where $N_{G}$ represents the number of genes in the gene set $G$.

The difference between the two empirical cumulative distribution functions is calculated for each gene in the gene set. The maximum difference across all the genes in the gene set is taken to be the enrichment score. A permutation-based $\mathrm{p}$-value is then calculated for each gene set which is used to identify significant alterations in expression across experimental conditions. A high enrichment score is achieved when a gene set contains a large number of highly ranked genes.

GSEA incorporates the magnitude of the gene fluorescence intensity values into its model. However, as discussed by Damian and Gorfine [11], GSEA is hindered by several factors. The primary concern is that the power of the test is a function of the number of genes in the pathway. Thus the method may not work well with small gene sets.

An alternative approach to examine pathway-associated effects is the Global test. This method was originally proposed to test correlation structures due to familial aggregation in pedigrees by Houwelingen et al. in 1995 [12,13]. This methodology applies a goodnessof-fit test for a generalized linear model having a canonical link function with a known dispersion parameter. In 2003, familial aggregation testing was adapted by Goeman et al. for microarray data analysis [8] and was designed to determine whether the common expression pattern of genes within a pre-defined set is significantly related to experimental condition. A generalized linear model is used to estimate a statistic for each gene set,

$$
\mu=E(Y \mid \beta)=h^{-1}\left(\alpha+\sum_{j=1}^{n} x_{i j} \beta_{j}\right) .
$$

where $Y$ represents the binary variable indicating the presence or absence of the treatment condition, $h$ is a link function, for example the logit function, and intercept $\alpha$. The $x_{i j}$ represents an element of the expression matrix of samples $i$ and genes $j$ and the regression coefficient $\beta_{j}$ for gene $j\left(j=1,2, \ldots, N_{G}\right)$. The Q-statistic for a gene set is given by

$$
Q=\frac{1}{\mu_{2}} \sum_{i=1}^{N_{G}} \sum_{j=1}^{N_{G}} R_{i j}\left(Y_{i}-\mu\right)\left(Y_{j}-\mu\right),
$$

where $\mathrm{R}$ is the covariance matrix of the expression data and $\mu_{2}$ is the second central moment of $Y$ under the null hypothesis. A high $Q$ value is achieved when at least one $\beta_{j}$ is significantly different than zero. However, the Global Test makes a distributional assumption that the regression coefficients are from the same normal distribution which is unlikely to be true.

In this paper we develop a rank based test, the Rank Test that takes into account the magnitude of the intensity value as well as the correlation between genes within a specific pathway. The advantage of these tests is that no assumptions on distribution or independence are made. Genes in a pathway are first aligned by subtracting the median expression value for the combined treatment and control groups. The aligned expression values are then ranked within each subject and the vector of average ranks is calculated for each treatment. The distance between the two treatments is calculated and a permutation analysis is used to obtain a p-value for each pathway. The R Code for the Rank Test will be made available upon request. We also investigate a restandardized version of the Rank Test (Modified Rank Test) where the observed distance is centered and scaled based on the mean and standard deviation from random subsets of genes of equal size.

Using two publicly available microarray datasets, we empirically evaluated Global and GSEA with the Rank Test and the Modified Rank Test. We also generated simulated data to test the reliability of each of the 
pathway analysis applications. Both real and simulated data were used to demonstrate that the rank based test has the highest, or nearly the highest power among the various techniques evaluated, especially when changes in the correlation structure of the pathway occurred. The rank based tests are robust and perform well under a wide range of assumptions.

\section{Results and Discussion \\ Data Description}

The Rank Test and the re-standardized Rank Test were compared with the Global test and with GSEA using two publicly available data sets. The first expression set is a mouse developmental toxicology experiment conducted by Dong et al. [14] using Agilent high-density oligonucleotide chips. The objective of the study was to investigate the effects of a thyroid disrupting chemical on the livers of developing pups. Pregnant dams were treated with 6-propyl-2-thiouracil (PTU) to produce hypothyroid pups. Livers were collected from control and PTU treated pups and RNA was labelled and hybridized to Agilent $22 \mathrm{~K}$ arrays against a universal mouse reference RNA. The expression data for this experiment are available from the European Bioinformatics Institute (EBI) repository (accession number E-MEXP-1091). In the second dataset, Halappanavar et al. [15] investigated the effects of mainstream tobacco smoke (MTS) on global transcription in the mouse lung. Male C57B1/CBA mice were exposed to MTS from two cigarettes per day, 5 days/week, for 6 or 12 weeks. Agilent high density DNA microarrays were used to characterize global gene expression changes in whole lung. The data were retrieved from the National Centre for Biotechnology Information (NCBI) database (accession number GSE12930). We used Agilent arrays in the present experiment because this is the technology that we use in our facility. However, the findings of this work, and the algorithms developed, should apply to data from other DNA microarray platforms using different probe technologies.

\section{Data from EBI: E-MEXP-1091}

In the Dong et al. [14] experiment, pregnant dams were rendered hypothyroid by treatment with $0.1 \%$ PTU in drinking water, from day 13 post-conception until weaning. Livers were collected from control and PTU treated pups at post-natal day (PND) 15. Each treatment group contained 5 males and 5 females. Treating gender as a block factor, we obtain two data sets, one for males and one for females. Analysis of the Agilent array consisted of 20651 probes which yielded 194 KEGG (Kyoto Encyclopedia of Genes and Genomes) pathways $[16,17]$. Pathways were constructed using the mgug4121a.db R library and consisted of only those pathways containing two or more genes with no missing expression values.

Using a MAANOVA [18] with an FDR corrected pvalue of 0.10 , Dong et al. [14] discovered 96 differentially regulated genes. Of these, 72 genes encoded proteins of known function. Approximately 50\% (34 genes) belonged to various metabolism pathways (as expected as liver is the primary site of metabolism). A second large group of genes were part of development pathways (10 genes), as expected as the treatment was delivered across a broad developmental time frame. Using ONTO express [19], Dong et al. [14] found the most affected biological processes included metabolism, cell growth and maintenance, development, immune response, transcription, and signal transduction. However, no specific KEGG pathways were presented in the paper as affected by the treatment.

We used the Dong et al. data to investigate the KEGG pathways that may be affected by the treatment. For the male and female expression sets, Global, GSEA, Rank and Modified Rank tests were applied to identify differences between the control and exposed groups for the 194 KEGG pathways. VENN diagrams using VENNY [20] were generated to compare the results within gender. The 0.01 significance level was chosen so that the Pre-Family Error Rate (PFE), i.e. the expected number of errors in the family, would be 1.94 for each method. Within males, a total of 41 pathways were significant, and 24 were unique to one of the 4 methods with only one common pathway (C21-Steroid hormone metabolism, Additional file 1: Table S1). Females exhibited 26 differential pathways, 12 of which were significant at the 0.01 level (Additional file 1: Table S1). The results for the male and female data are summarized in Figures 1 and 2 .

Assuming no gender differences with respect to the identified pathways, VENN diagrams were generated. The striking observation is that GSEA had no common differential pathways for males and females, where the Global, Rank and Modified Rank methods had 7, 9, and 6 common pathways respectively. Of the 9 common pathways for the Rank test five contained at least one gene that was validated by RT-PCR in the Dong et al. study. For the Global method two of the seven pathways contained genes that were validated. For the Modified Rank test four of the six pathways contained at least one gene that was validated. Thus, a fair proportion of these pathways contain at least one gene validated by an alternative technology, providing some degree of confidence in the involvement of these pathways in response to PTU exposure.

The Endocrine signalling pathway, labelled GnRH was in Top 5 pathways ranked by p-value for GSEA in the male results. The liver is not the site of $\mathrm{GnRH}$ 


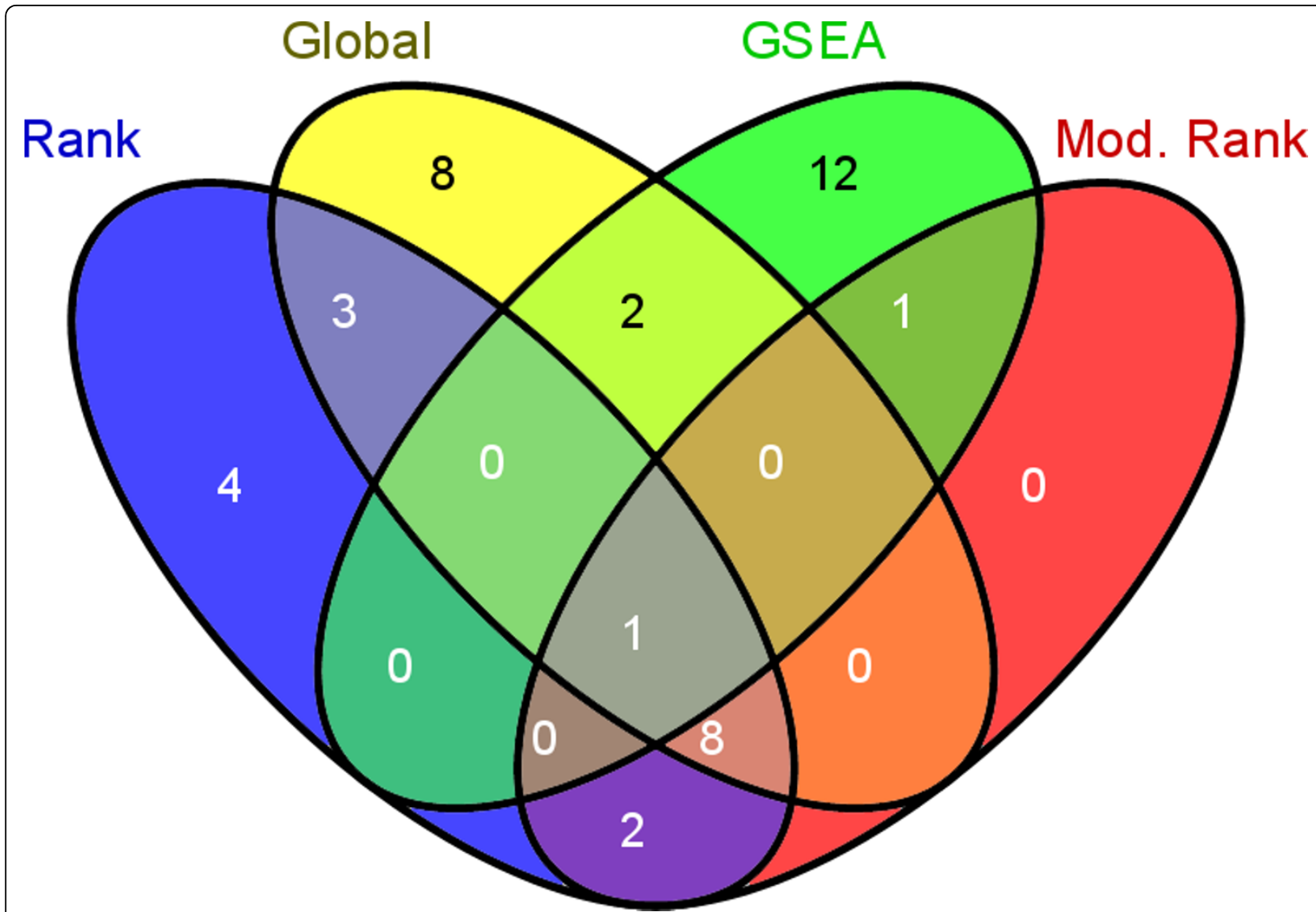

Figure 1 Summary of the results from the male data for the 4 methods.

production and thus we don't expect that this pathway is affect per se. However, many of the genes that are found in this pathway are involved in numerous other signal transduction pathways. For example, the gene Egfr (epidermal growth factor receptor) is part of 12 other KEGG pathways, Protein Kinase C (Prkca) is a node in 23 other KEGG pathways, Ras is part of 20 (not including pathways related to human disease), Jun is part of 9, and various genes in the GnRH pathway that were differentially expressed are part of MAPK signalling pathways. This, in combination with the lack of change in the GnRH receptor itself, suggest that this pathway was found because of a broad level of change in signal transduction pathways, rather than a direct effect on GnRH signalling. Thus, the identification of this pathway is a good example demonstrating that responsive pathways need to be carefully scrutinized and biologically validated.

We generally observed two types of differences between control and treatment groups that affected gene rankings within pathways. Most gene set methods detected changes in mean intensities. For the C21-Steroid hormone metabolism pathway (Figure 3) of the 13 genes in the pathway, Akr1c18 and Hsd3b1 have fold changes less than -2.5 for both genders and for the males, Hsd11b1 and Hsd3b2 have fold changes greater than 1.6. This pattern implies that rankings of genes in these pathways diverge between control and treatment groups. However, the changes in this pathway in the female dataset were not of sufficient magnitude to be found significant by the GSEA test. Previous work has shown a significant increase in progesterone in the plasma of female rats that were rendered hypothyroid by chemical treatment (e.g., Tohei [21]). The primary gene involved in the maintenance of progesterone homeostasis is Akr1c18 (also known as 20-alpha-hydroxysteroid dehydrogenase). Dong et al.'s data indicate a 6-7 fold down-regulation in both males and females for this gene in hypothyroid livers of developing mice (Figure 1). Akr1c18 is specific to the C21 steroid hormone metabolism pathway. The other gene that shows consistent down-regulation in both sexes is 3-beta-hydroxydelta 5 -steroid dehydrogenase (Hsd3b1/Hsd3b2), which also participates in the maintenance of progesterone homeostasis. Additional genes in the C21 steroid hormone metabolism pathway are altered by the treatment, 


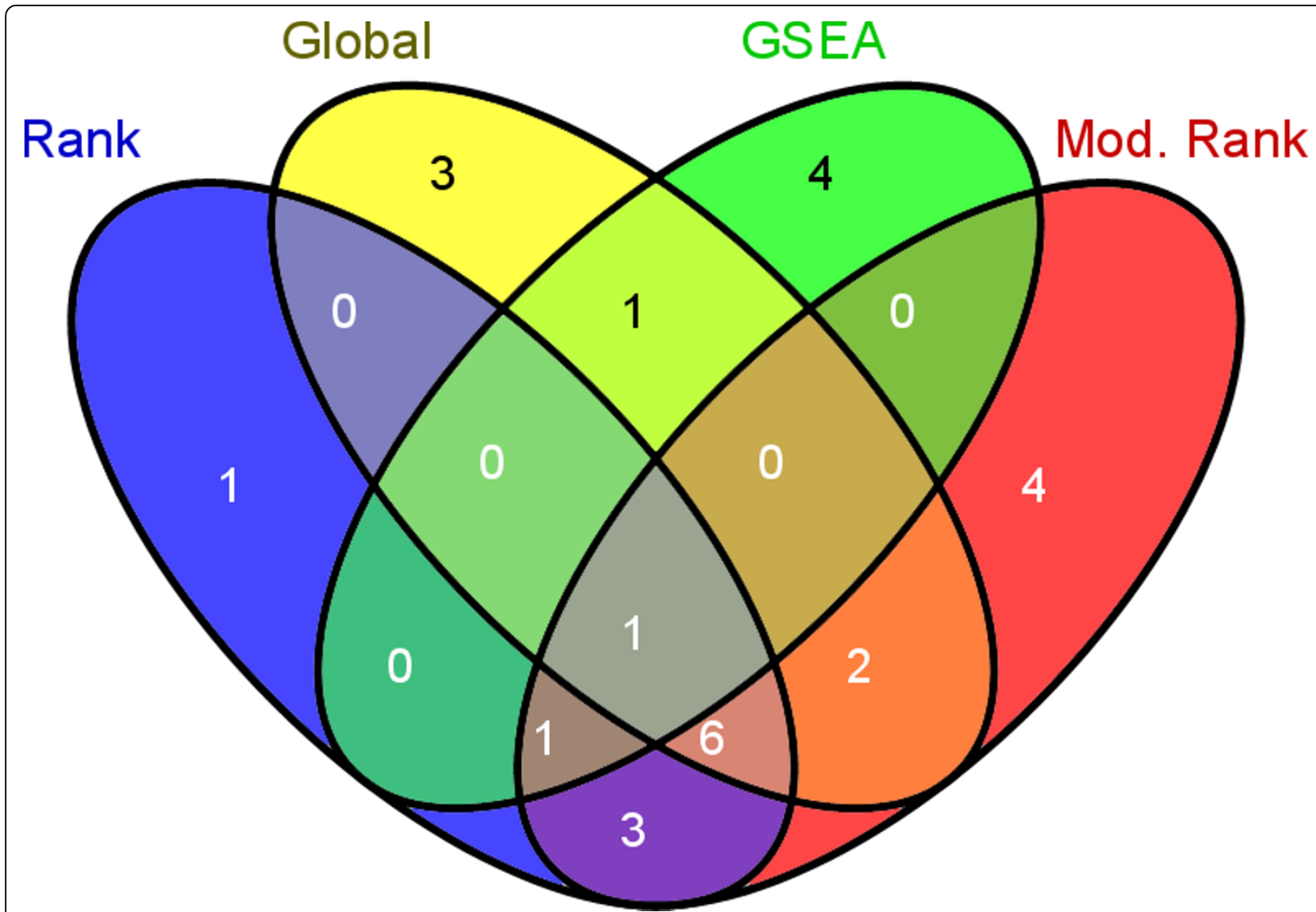

Figure 2 Summary of the results from the female data for the 4 methods.

but show smaller fold changes or exhibit differences between males and females. However, two strong hits in this pathway in combination with published evidence in the literature demonstrating disruptions in progesterone levels as a result of altered thyroid hormone levels provide evidence to suggest that genes operating in this pathway are affected by the treatment either directly or indirectly in the livers of developing mice. Thus, we argue that the GSEA may have failed to identify C21 steroid hormone metabolism as an affected pathway.

A second type of difference between groups is due to change in the correlation between genes within a pathway. To examine the correlation between genes, the ranks of relative expression for each gene is established. Any two genes can be positively or negatively correlated or not correlated. One would expect that genes within a pathway to be correlated. If there is a shift in these correlations as a result of treatment this may affect this relationship among the genes. One example is the alpha-Linolenic acid metabolism pathway (Figure 4). The distribution of gene correlations for the control samples has two modes approximately at -0.5 and at 0.4 . The distribution of gene correlations for the treated samples is different having a single mode close to 0 . This suggests that there are more genes that are now correlated compared to the control distribution. The Rank Test identified a significant difference for the male and female datasets and the Global Test identified a significant difference for the males, but the other statistics failed to detect a significant difference. In both genders, Fads2 was greater than 2 fold down-regulated and Pla2g12a was greater than 1.5 up-regulated. Also, Acox1 for males was greater than 1.5 up-regulated. In terms of changes in correlations, Pla2g2c and Pla2g12b for the males samples had a spearman correlation of -0.8 for the controls and 0.6 for the treated samples, whereas for the females genes Pla2g4a and Pla2g2e had correlations of -0.9 and 0.8 for treated and controls. In all, there were 13 of 91 comparisons that had differences in correlation greater than 1.4. The distribution of correlations for the females also exhibited a similar pattern as in Figure 4 providing additional validation for disruption of this pathway. Disruptions in TH levels are known to result in alterations in fatty acid metabolism. A relationship between $\mathrm{TH}$ and alpha linolenic acid in the liver has previously been established $[22,23]$. Thus, the 


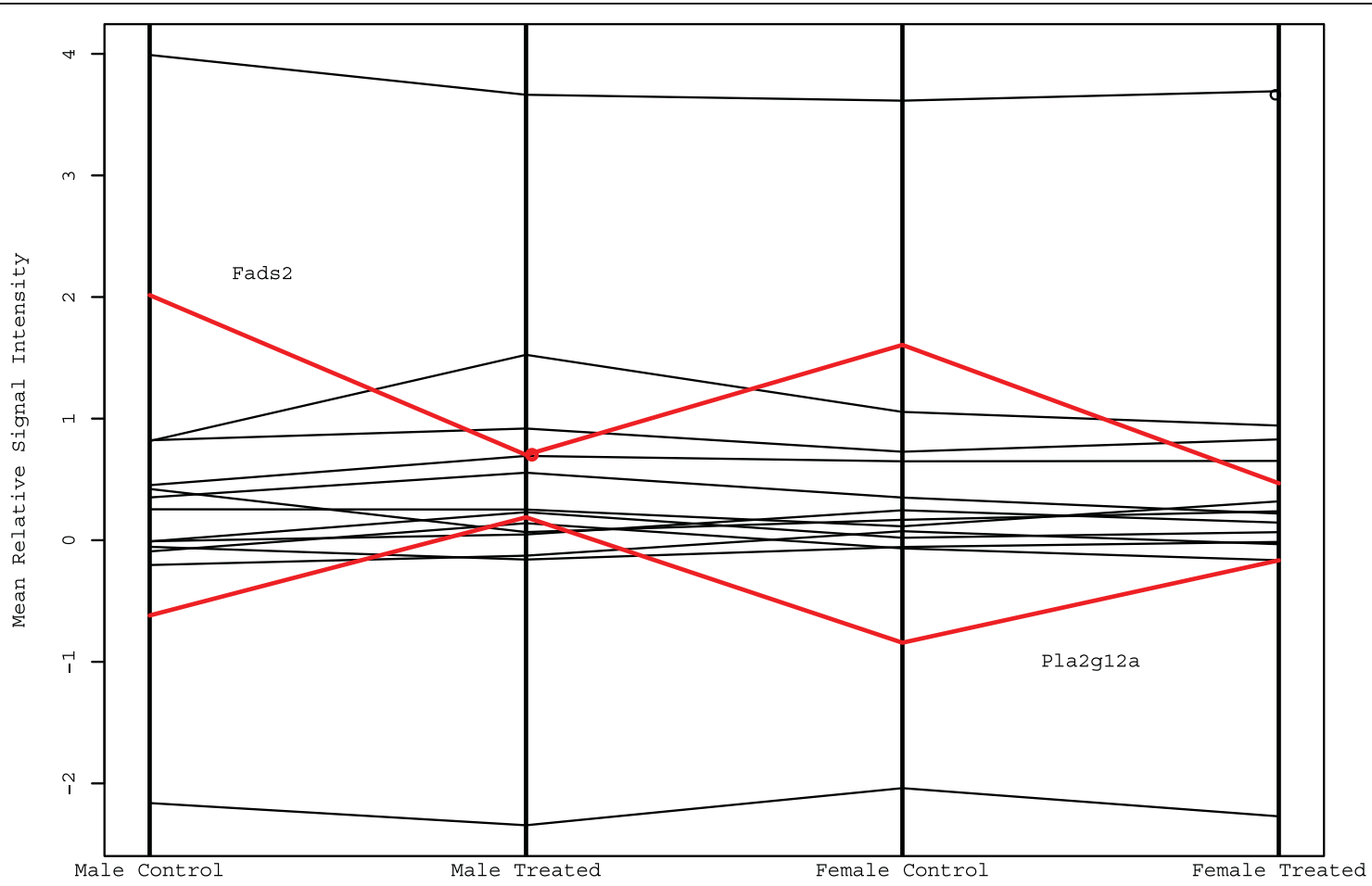

Figure 3 C21-Steroid hormone metabolism pathway. Parallel co-ordinate plots of the genes in the C21-Steroid hormone metabolism pathway are displayed. The genes Akr1c18 and Hsd3b1 are identified by red lines.

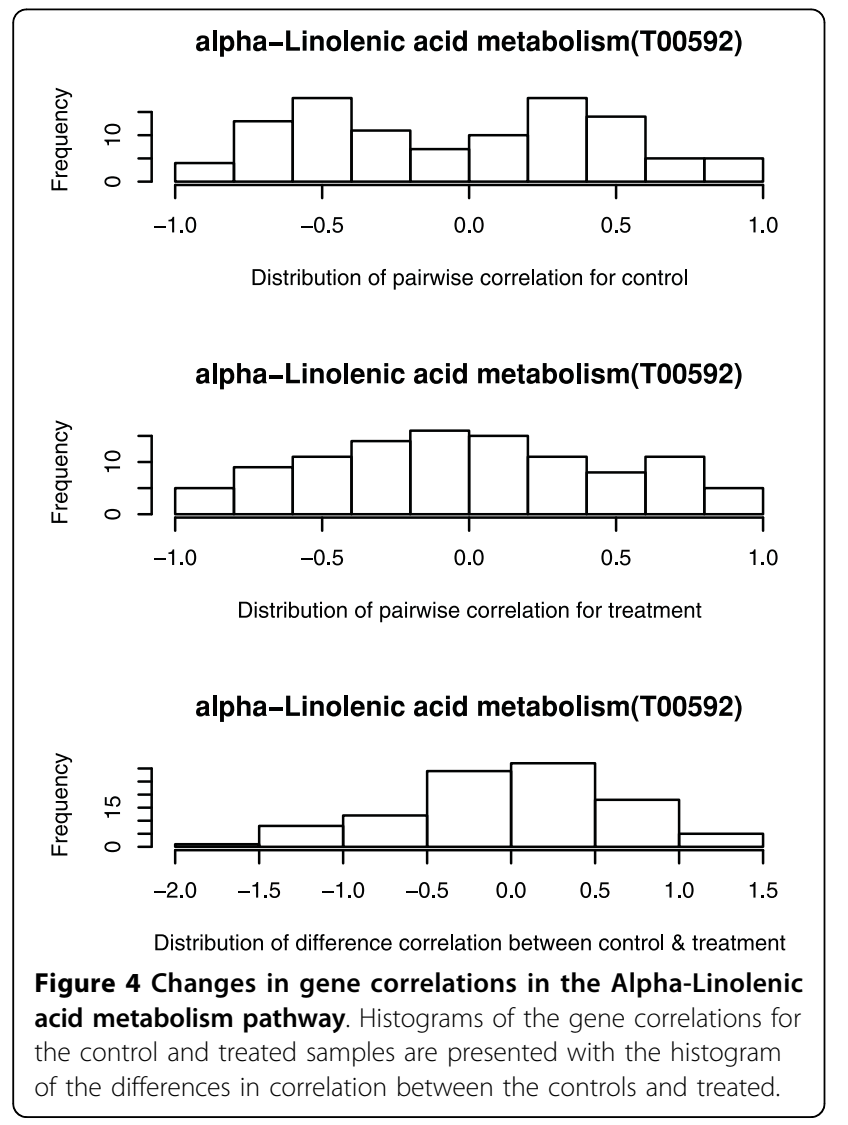

identification of this pathway is likely to be biologicallyrelevant.

The aim of the test that we have developed is to generate hypotheses based on microarray experimental data to assist in prioritizing follow-up experiments. Without extensive biological validation it is not possible to comment on whether these pathways have been correctly identified. Thus, we argue that the above pathways may be promising candidates for additional research. We describe one further biological investigation below, but a more powerful validation exercise is the simulation analysis described in the last section of this paper.

\section{Data from NCBI: GSE12930}

Halappanavar et al. [15] used DNA microarrays to examine global transcriptional changes in whole lung tissues derived from mice exposed to mainstream tobacco smoke (MTS) for 6 or 12 weeks. From the MAANOVA analysis, 79 genes were either up- or down-regulated following MTS exposure. Among the 79 statistically differentially expressed genes cytochrome P450, family 1 (Cyp1a1), heme oxygenase (decycling) 1 (Hmox1) and $\mathrm{NAD}(\mathrm{P}) \mathrm{H}$ dehydrogenase, quinine 1 (Nqo1) were validated using RT-PCR. In addition, cytokine interleukin 6 (IL-6) mRNA was upregulated alongside its antagonist, suppressor of cytokine signalling (SOCS3). These genes had also been reported in 
previous smoke-exposure studies. The authors examined IL-6 protein levels to confirm the finding, as well as some of its downstream targets. In this study, Pathway Studio (version-5, Ariadne Genomics Inc.) was used to identify pathways. From their analysis they identified a network of two core modules relating to the xenobiotic response pathway, and inflammation, cell survival and proliferation pathway.

The pathways identified at the 0.01 significance level by either the Rank Test, Global Test, GSEA and the Modified Rank test for the six week exposure were also identified by the respective methods in the twelve week exposure (Additional file 2: Table S2), with the exception of four pathways (Global: Basal transcription factors; Modified Rank Test: Glutamate metabolism, PPAR signalling pathway and Glioma). The Global test was the most sensitive statistic identifying 7 and 22 significant pathways followed by the Rank Test with 1, 17 and the Modified Rank Test with 6 and 11 significant pathways for the six and twelve week exposure respectively. The GSEA method did not identify any significant pathways. Of the 29 pathways declared significant, five pathways (Metabolism of xenobiotics by cytochrome P450, Tryptophan metabolism, Porphyrin and chlorophyll metabolism, Biosynthesis of steroids and Arachidonic acid metabolism) contained at least 1 gene that was RT-PCR validated in the Halappanavar et al. [15]. The gene Ptgs2 in the Arachidonic acid metabolism pathway was the only pathway that was unique to one method, the Global Test.

Thus, the general findings of the reanalysis of these publicly-available experimental data provide compelling support to the advantages of rank and global methods. However, without extensive RT-PCR validation, it is not possible to know if these methods identify more pathways, or are more accurate than the GSEA approach. As such, in the following sections we apply a simulation experiment to test these statistics.

\section{Simulation}

The issues with empirical evaluations of methods are that we often do not know the underlying distributions of the data, i.e., the truth. Simulation is a tool often used to test new methodologies. Simulating data from known distributions allows us to measure how one method performs compared to another method under different scenarios.

The simulation conducted in this paper provides further evidence to support the ranking approach as a more sensitive method. In the proceeding sections we demonstrated that when the treatment and control samples come from the same population, the power of the rank test is higher. As well, we will evaluate the methods with respect to their ability to detect differences in mean, correlation or both.

For this simulation study only one pathway was generated. The number of probes in the pathway was set to 46 genes which is the average pathway size based on the E-MEXP-1091 dataset. Control samples were generated from a standard multivariate normal distribution $\mathrm{N}(0, \mathrm{I})$. We considered a variety of changes to characterize the treatment groups:

1) Mean change: the data were generated from the same distribution as the control samples except that the mean of the first three genes of the pathway was set to equal 2;

2) Correlation change: the data were simulated from a multivariate normal distribution $\mathrm{N}(0, \Sigma)$. The covariances between gene pairs in a pathway were set equal to 0.9 and the variances equal to 1 ;

3) Correlation and mean change.

For each of the above conditions, sample sizes of 5 , 20 , and 30 per group were used. The simulation was conducted as follows:

1) Data for the control and treated conditions for a given scenario and sample size were generated and then centered using the median.

2) The GSEA, Global and Rank Test tests were applied and p-values were obtained using 1000 random permutations (note that here the Modified Rank Test is equivalent to the Rank Test).

3) Significance was determined if the p-value from the test was less than or equal to 0.1 .

For each scenario and sample size the above procedure was repeated 1000 times and the estimated power for each test was recorded in Table 1. A sample size of five was chosen as this is a typical sample size for most microarray experiments, while 20 and 30 replicates provide large sample properties of the statistic.

All three methods provide an error rate of approximately 0.1 in the no change case. The mean change condition the Global Test had the largest observed power $(0.853)$ for a sample size of 5 , followed by the Rank Test with a power of 0.739 . The Rank and Global Test converged to a power of 1 at the 20 and 30 sample size. The GSEA method had the lowest power for this test case. Under the correlation change case, the Rank test out-performed the Global and GSEA methods. The Rank test achieved a power of 0.972 for the small sample size case whereas the other two methods had power estimates that resemble the no change test case for the small and large sample scenarios. When changes in the mean and correlation occurred, the power for the Rank 
Table 1 Power calculations from the simulation study

\begin{tabular}{llccc}
\hline & & \multicolumn{3}{c}{ Sample Sizes per Group } \\
\cline { 3 - 5 } Simulated Difference & Method & $\mathbf{5}$ & $\mathbf{2 0}$ & $\mathbf{3 0}$ \\
\hline No Change & Rank & 0.117 & 0.102 & 0.095 \\
& Global & 0.107 & 0.051 & 0.056 \\
& GSEA & 0.092 & 0.120 & 0.112 \\
\hline Mean Change & Rank & 0.739 & 1.000 & 1.000 \\
& Global & 0.853 & 1.000 & 1.000 \\
& GSEA & 0.230 & 0.777 & 0.950 \\
\hline Correlation Change & Rank & 0.972 & 0.997 & 1.000 \\
& Global & 0.131 & 0.102 & 0.107 \\
& GSEA & 0.119 & 0.095 & 0.109 \\
\hline Mean and Correlation Change & Rank & 1.000 & 1.000 & 1.000 \\
& Global & 0.422 & 0.993 & 1.000 \\
& GSEA & 0.075 & 0.053 & 0.050 \\
\hline
\end{tabular}

Presented is the estimated power of the test for each method at varying sample sizes.

Test was 1 for all sample sizes. The Global test outperformed GSEA and the power of the test approached 1 with increasing sample size. GSEA did not perform well when changes in correlation occurred, obtaining power estimates one would expect by chance. Considering the simulated conditions and sample sizes used the Rank Test was the most powerful test.

\section{Conclusions}

Dimension reduction is critical in order to decipher underlying biological phenomena in microarray studies. Gene sets based on pathways or GO ontology provide an ideal approach to reduce the dimensionality through biological meaning. Working with pathways and gene sets that cover the probe sets on the microarray platform, rather than the individual probes, can dramatically reduce dimensionality and aid in biological interpretation. Using this approach, the investigator can evaluate datasets more readily compared to interpreting potentially long lists of differentially expressed genes.

We describe a non-parametric methodology to test whether or not a pathway exhibits a significant change compared to a control. The method revealed a large number of significantly changed pathways that were identified more efficiently and potentially more accurately than can be achieved by manually mining gene lists derived from standard analyses. Results for interesting pathways from this method are not impacted by their environments or surroundings, which happen in other existing methods (e.g. Fisher Exact's Test and GSEA). Moreover, our method takes into account changes in both the mean and in the correlation. The Rank and Modified Rank test demonstrate good performance on real as well as on simulated microarray data sets. However, the low degree of overlap between the approaches suggests that the use of more than one technique may be beneficial when conducting analyses of gene sets to avoid missing a novel result. In addition, the investigator is urged to continue to use alternative technologies (e.g., RT-PCR, protein analysis, etc.) to validate findings.

\section{Methods \\ Normalization}

For the empirical evaluation using the E-MEXP-1091 and GSE12930 datasets, the lowess approach [24] was used to normalize the data. Per-gene normalization was then performed centering the expression data by the median. Analysis was carried out on all genes regardless of flags.

\section{GSEA and Global}

All analysis was conducted in R [25]. The Bioconductor [26] library and the GSEA $1.0 \mathrm{R}$ package [5] were used. For the Global methodology, the Global test function in the Global test library was used to identify significant pathways.

\section{Rank Test}

Assume that there are $M$ genes belonging to a pathway. Subtract from each gene expression value, the median expression value obtained from the combined treatment and control groups. This process aligns the data thereby inducing subsequent analyses to be sensitive to changes in the mean. Next, for the $j^{\text {th }}$ subject in group $i$, let $\omega_{i j}$, represent the vector of ranks of the aligned intensity values of the $M$ genes in the pathway. Set

$$
m_{C}-m_{T}=\frac{1}{n_{1}} \sum_{j=1}^{n_{1}} \omega_{j 1}-\frac{1}{n_{2}} \sum_{j=1}^{n_{2}} \omega_{j 2}
$$

The use of ranks serves two purposes. First, it captures for each subject, the correlation pattern of the aligned expression values. Second, it allows for a subsequent nonparametric analysis.

Motivated by the methods of Feigin and Alvo [27], we propose the test statistic

$$
S=\left[m_{C}-m_{T}\right]^{\prime}\left[m_{C}-m_{T}\right]
$$

where prime indicates the transpose of the vector. Under the hypothesis that there is no change between the two groups, the statistic $S$ should be small in magnitude. Let $S_{o b s}$ be the value of the observed statistic.

Next, we propose a permutation test based on $S$. Under the null hypothesis that no change has occurred, the subjects in the two groups are interchangeable. Hence, we compute for each selection of $n_{1}$ subjects 
from $n$ a value of the statistic $S$. The nominal p-value is then given as

$$
p-\text { value }=\frac{\#\left[S \geq S_{\text {obs }}\right]}{\left(\begin{array}{c}
n \\
n_{1}
\end{array}\right)}
$$

When the total number of possible permutations $\left(\begin{array}{c}n \\ n_{1}\end{array}\right)$ is large, we randomly choose 1000 permutations among them.

\section{Modified Rank Test}

The Rank test is defined independently of the other genes contained in the microarray. Efron and Tibshirani [28] considered two different hypotheses in connection with the problem of assessing the statistical significance of a pathway. The random null hypothesis states that the $M$ genes in the pathway of interest have been chosen at random from the array. Hence, the null distribution of the test statistic is obtained by considering its value over all the possible sets of $M$ genes in the array. On the other hand, to each subject corresponds an $M$ vector of expression values. The permutation hypothesis in that case states that the vectors are independent and identically distributed and hence, the distribution of the test statistic is obtained by permuting the vectors. As Efron and Tibshirani [28] point out, both hypotheses have shortcomings. The first tends to ignore correlations among the genes whereas the second does not take into account the array from which the genes are drawn. Instead, they proposed an adjusted statistic which restandardizes the observed statistic $S_{o b s}$ with mean $m^{*}$ and standard deviation $\sigma^{*}$ as follows:

$$
S^{*}=m^{*}+\sigma^{*}\left(\frac{S_{o b s}-m_{S}}{\sigma_{S}}\right)
$$

where $m^{*}, \sigma^{*}$ are the mean and standard deviation obtained by randomly selecting gene sets from the entire microarray and $m_{s}$ and $\sigma_{\mathrm{s}}$ are the mean and standard deviation obtained by permutation of the labels for the specific pathway.

Additional file 1: Table 1 Significant Pathways identified in the EMEXP-1091 Data sets. A pathway is considered significant if the $p$-value of the test statistic is less than or equal to 0.01 .

Click here for file

[http://www.biomedcentral.com/content/supplementary/1471-2105-1160-S1.XLS]

Additional file 2: Table 2 Significant Pathways discovered in GSE12930 Data Sets. A pathway is considered significant if the p-value of the test statistic is less than or equal to 0.01 . Click here for file [http://www.biomedcentral.com/content/supplementary/1471-2105-1160-S2.XLS]

\section{Acknowledgements}

We would like to acknowledge Mike Wade, and Morie Malowany for helpful discussions and comments on the manuscript.

\section{Author details}

'Department of Mathematics and Statistics, University of Ottawa, Ottawa, ON K1N 6N5 Canada. ${ }^{2}$ Environmental Health Science and Research Bureau, Environmental and Radiation Health Sciences Directorate, Health Canada, Ottawa ON K1A OK9 Canada.

\section{Authors' contributions}

The methodology for the proposed Rank Statistic was developed by MA. All analysis and code development was conducted by ZL. The biological interpretation of the case studies evaluated was conducted by $C Y$. The manuscript was drafted and prepared by MA, ZL, AW and CY. MA was the overall team leader. All authors have read and approved the final manuscript.

Received: 27 October 2009

Accepted: 28 January 2010 Published: 28 January 2010

\section{References}

1. Khatri P, Draghici S, Ostermeier G, Krawetz S: Profiling gene expression using onto-express. Genomics 2002, 79(2):266-270.

2. Draghici S, Khatri P, Martins R, Ostermeier G, Krawetz S: Global functional profiling of gene expression. Genomics 2003, 81(2):98-104.

3. Draghici S, Khatri P, Tarca A, Amin K, Done A, Voichita C, Georgescu C, Romero R: A systems biology approach for pathway level analysis. Genome Research 2007, 17(10):1537.

4. Mootha V, Lindgren C, Eriksson K, Subramanian A, Sihag S, Lehar J, Puigserver P, Carlsson E, Ridderstraale M, Laurila E, Houstis N, Daly MJ, Patterson N, Mesirov JP, Golub TR, Tamayo P, Spiegelman B, Lander ES, Hirschhorn JN, Altshuler D, Groop LC: PGC-1-responsive genes involved in oxidative phosphorylation are coordinately downregulated in human diabetes. Nature Genetics 2003, 34(3):267-273.

5. Subramanian A, Tamayo P, Mootha V, Mukherjee S, Ebert B, Gillette M, Paulovich A, Pomeroy S, Golub T, Lander E, Mesirov JP: Gene set enrichment analysis: A knowledge-based approach for interpreting genome-wide expression profiles. Proceedings of the National Academy of Sciences 2005, 102(43):15545-15550.

6. Barry W, Nobel A, Wright F: Significance analysis of functional categories in gene expression studies: a structured permutation approach. Bioinformatics 2005, 21(9):1943-1949.

7. Tian L, Greenberg S, Kong S, Altschuler J, Kohane I, Park P: Discovering statistically significant pathways in expression profiling studies. Proceedings of the National Academy of Sciences 2005, 102(38):13544-13549.

8. Goeman J, Geer van de S, de Kort F, van Houwelingen $\mathrm{H}$ : A global test for groups of genes: testing association with a clinical outcome. Bioinformatics 2004, 20:93-99.

9. Kong S, Pu W, Park P: A multivariate approach for integrating genomewide expression data and biological knowledge. Bioinformatics 2006, 22(19):2373

10. Liu Q, Dinu I, Adewale A, Potter J, Yasui Y: Comparative evaluation of gene-set analysis methods. BMC Bioinformatics 2007, 8:431.

11. Damian D, Gorfine M: Statistical concerns about the GSEA procedure. Nature Genetics 2004, 36(7):663.

12. le Cessie S, van Houwelingen $\mathrm{H}$ : Testing the Fit of a Regression Model Via Score Tests in Random Effects Models. Biometrics 1995, 51(2):600-614.

13. Houwing-Duistermaat J, Derkx B, Rosendaal F, van Houwelingen H: Testing Familial Aggregation. Biometrics 1995, 51(4):1292-1301.

14. Dong H, Yauk CL, Williams A, Lee A, Douglas GR, Wade MG: Hepatic gene expression changes in hypothyroid juvenile mice: Characterization of a novel negative thyroid responsive element. Endocrinology 2007, en.20070452. 
15. Halappanavar S, Russell M, Stampfli MR, Williams A, Yauk CL: Induction of the interleukin 6/signal transducer and activator of transcription pathway in the lungs of mice sub-chronically exposed to mainstream tobacco smoke. BMC Medical Genomics 2009, 2:56.

16. Kanehisa M: A database for post-genome analysis. Trends in Genetics 1997, 13(9):375-376

17. Kanehisa M, Goto S: KEGG: Kyoto encyclopedia of genes and genomes. Nucleic acids research 2000, 28:27.

18. Wu H, Kerr M, Cui X, Churchill G: MAANOVA: a software package for the analysis of spotted CDNA microarray experiments. The analysis of gene expression data: methods and software 2003, 323-341.

19. Draghici S, Khatri P, Bhavsar P, Shah A, Krawetz S, Tainsky M: Onto-tools, the toolkit of the modern biologist: onto-express, onto-compare, ontodesign and onto-translate. Nucleic acids research 2003, 31(13):3775.

20. Oliveros J: VENNY. An interactive tool for comparing lists with Venn Diagrams. 2007

21. Tohei A: Studies on the functional relationship between thyroid, adrenal and gonadal hormones. J Reprod Dev 2004, 50(1):9-20, Review.

22. Hoch FL: Lipids and thyroid hormones. Prog Lipid Res 1988, 27:199-270

23. Raederstorff $D$, Meier $C A$, Moser $U$, Walter $P$ : Hypothyroidism and thyroxin substitution affect the $\mathrm{n}-3$ fatty acid composition of rat liver mitochondria. Lipids 1991, 26(10):781-7.

24. Yang YH, Dudoit S, Luu P, Lin DM, Peng V, Ngai J, Speed TP: Normalization for CDNA microarray data: a robust composite method addressing single and multiple slide systematic variation. Nucleic Acids Res 2002, 30:e15.

25. Team R: R: A language and environment for statistical computing Vienna, Austria: R Foundation for Statistical Computing 2008.

26. Gentleman R, Carey V, Bates D, Bolstad B, Dettling M, Dudoit S, Ellis B, Gautier L, Ge Y, Gentry J, Hornik K, Hothorn T, Huber W, lacus S, Irizarry R, Leisch F, Li C, Maechler M, Rossini AJ, Sawitzki G, Smith C, Smyth G, Tierney L, Yang JY, Zhang J: Bioconductor: open software development for computational biology and bioinformatics. Genome Biology 2004, 5(10):R80.

27. Feigin P, Alvo M: Intergroup Diversity and Concordance for Ranking Data: An Approach via Metrics for Permutations. The Annals of Statistics 1986, 14(2):691-707.

28. Efron B, Tibshirani R: On testing the significance of sets of genes. Ann App/ Stat 2007, 1(1):107-129.

doi:10.1186/1471-2105-11-60

Cite this article as: Alvo et al: Testing for mean and correlation changes in microarray experiments: an application for pathway analysis. $B M C$ Bioinformatics 2010 11:60

\section{Submit your next manuscript to BioMed Central and take full advantage of:}

- Convenient online submission

- Thorough peer review

- No space constraints or color figure charges

- Immediate publication on acceptance

- Inclusion in PubMed, CAS, Scopus and Google Scholar

- Research which is freely available for redistribution

Submit your manuscript at www.biomedcentral.com/submit
Biomed Central 